Helgoländer wiss. Meeresunters. 17, 398-410 (1968)

\title{
Veränderungen des Meeres durch Abfälle und die darauf basierende Einführung von fünf Gefahrenklassen für das Einbringen von Abfällen ins Meer
}

\author{
GÜNTER WEICHART \\ Deutsches Hydrographisches Institut, Hamburg
}

\begin{abstract}
Changes of the sea by wastes, and the introduction of five danger classes for the disposal of wastes in the sea, based on such changes. There are four different kinds of changes caused by the disposal of wastes into the sea: changes on the sea floor, in the seawater, at the surface and in the air above the sea. The measure for the harmful effect of insoluble wastes on the sea floor is the area covered by the wastes. The measure for harmful effects in sea-water is the "water volume with harmful properties"; this is the volume in which a certain critical value (temperature, concentration, etc., e. g. in regard to the tolerance limits of organisms) is surpassed. In case of continuous waste disposal, the constant value (with time) of the water volume with harmful properties is decisive; in case of a singular or repeated disposal, the maximum value (with time). The maximum value of the water volume with harmful properties is almost equal to the water volume necessary for making a waste harmless by dilution. It is necessary to keep negative effects of wastes on organisms, fisheries, sport and tourism, and - last but not least - on the health of mankind, as small as possible. Keeping this necessity in mind, a classification of wastes and sea areas is proposed. This classification divides the wastes, according to substance and quantity, into five classes and provides information whether or not wastes may be disposed of in the sea and at which depths. Special criteria are provided for the classification of waste products; these criteria take into consideration type, extent, and duration of the changes caused in the sea. Examples are given to demonstrate the application of the proposed classification in praxis. Criteria for waste classification and instructions for disposal in sea areas of different depths are given in a table.
\end{abstract}

\section{EINLEITUNG}

Der Begriff „Abfall“ wird hier für sämtliche Abfallstoffe und Abwässer sowie für Abfallwärme etc. verwendet. Ein Abfall sei hier nicht nur durch den Stoff, sondern auch durch die Menge charakterisiert.

Das Einbringen von Abfällen ins Meer läßt sich nicht generell befürworten oder ablehnen. Es Irommt nämlich wesentlich darauf an, welche physikalischen, chemischen und physiologischen Eigenschatten die ins Meer einzubringenden Abfälle besitzen und wie groß die insgesamt und die pro Zeiteinheit einzubringenden Abfallmengen sind. Je nach diesen Gegebenheiten können die Wirkungen verschiedener Abfälle auf die Lebewesen des Meeres und der Küstengebiete sowie auf Schiffahrt, Fischerei, 
Sport, Tourismus und Gesundheit des Menschen sehr unterschiedlich sein. Unter Berücksichtigung aller möglichen Wirkungen wird man unterscheiden zwischen solchen Abfällen, die unter keinen Umständen ins Meer eingebracht werden dürfen und solchen, deren Einbringung an bestimmten Stellen des Meeres unter bestimmten Bedingungen gerechtfertigt erscheint.

\section{VERANDERUNGEN DES MEERES DURCH ABFALLE}

Es gibt verschiedene Arten von Abfällen, beispielsweise kompakte Abfallstoffe, Abwässer, Abfallwärme etc. Abfallstoffe können die verschiedensten Eigenschaften besitzen:

Sie können fest, flïssig oder gasförmig sein. Sie können schwerer oder leichter als Meerwasser sein. Sie können leicht löslich oder schwer löslich in Meerwasser sein. Sie können flüchtig oder nicht flüchtig sein. Sie können mit dem Meerwasser chemisch reagieren. Sie können durch Licht, Bakterien etc. chemisch verändert werden. Sie können durch Meeresorganismen angereichert werden. Sie können giftig oder ungiftig sein.

Entsprechend ihren Eigenschaften können Abfälle im Meer und in der darüber befindlichen Luft sehr verschiedene Veränderungen hervorrufen. Nach dem Ort ihres Auftretens kann man vier Arten von Veränderungen unterscheiden: Veränderungen am Meeresboden, im Meerwasser, an der Meeresoberfläche und in der Luft über dem Meer.

\section{Veränderungen am Meeresboden}

Schwerlösliche Abfallstoffe, die dichter als Meerwasser sind, zum Beispiel Eisenoxide, können sich am Meeresboden ablagern. Außerdem können Abfallstoffe, die sich zunächst in Lösung befinden, durch chemische Reaktion mit dem Meerwasser ausfallen und zu Boden sinken. Auf diese Weise wird am Meeresboden eine bestimmte Fläche mit Abfallstoffen zugedeckt. Diese Fläche kann als Maß für die Schädlichkeit eines auf den Meeresboden versenkten, schwerlöslichen Abfalls dienen.

\section{Veränderungen im Meerwasser}

Die bedeutendsten Veränderungen des Meeres durch Abfälle sind die Veränderungen des Meerwassers. Unter diesem sind wiederum die chemischen Veränderungen durch lösliche Abfallstoffe die weitaus wichtigsten. Demgegenüber treten die physikalischen Veränderungen des Meerwassers, wie beispielsweise die Erhöhung der Temperatur durch Abfallwärme, in den Hintergrund.

Das Einbringen löslicher Abfallstoffe ins Meer bewirkt im allgemeinen Konzentrationserhöhungen. Gleichzeitig können aber auch Konzentrationsverminderungen eintreten, wenn Abfallstoffe mit den Bestandteilen des natürlichen Meerwassers che- 
misch reagieren. So kann beispielsweise die Sauerstoff-Konzentration des Meerwassers durch das Einbringen von Fäkalien oder Nitrit verringert werden.

Das Einbringen von löslichen Abfallstoffen ins Meer kann nicht nur eine Konzentrationsänderung der bereits im natürlichen Meerwasser vorhandenen Stoffe bewirken. Vielmehr können durch die Abfälle auch solche Stoffe ins Meer gelangen, die im natürlichen Meerwasser nicht vorhanden sind, zum Beispiel Pestizide, Phenole, Cyanide etc.

Die Veränderungen des Meerwassers, die durch das Einbringen von Abfällen hervorgerufen werden, können verschieden stark sein. Wird durch das Einbringen eines Abfalls ein bestimmter Grenzwert, zum Beispiel eine Grenztemperatur oder eine Grenzkonzentration, über- oder unterschritten, so können Schäden auftreten. Beispielsweise kann die Grenzkonzentration eines Giftstoffes überschritten oder die Grenzkonzentration des Sauerstoffs im Meerwasser unterschritten werden.

Die Festlegung der Grenzwerte kann nach verschiedenen Gesichtspunkten vorgenommen werden. In den weitaus meisten Fällen wird man sich nach der Erträglichkeit für Organismen richten. In diesen Fällen ist dann der Grenzwert mit der Erträglichkeitsgrenze identisch. Bei bestimmten Abfallstoffen, z. B. bei Schwermetallen, muß man auch die Anreicherung in der Nahrungskette mit berücksichtigen. Es gibt aber auch andere Gesichtspunkte, die für die Festlegung von Grenzwerten ausschlaggebend sein können, beispielsweise die Störung von Seewasserentsalzungsprozessen und die Zerstörung von Bauwerken oder Schiffen aus Holz oder Metall.

Bei kontinuierlichem Einbringen eines Abfalls befindet sich im stationären Zustand um die Einlaßstelle herum ein zeitlich konstantes Meerwasservolumen, in dem ein bestimmter Grenzwert über- oder unterschritten ist. Dieses Meerwasservolumen wollen wir als „schädlich verändert" bezeichnen. Das schädlich veränderte Meerwasservolumen ist ein sehr gutes Maß für die Schädlichkeit eines kontinuierlich ins Meer eingebrachten Abfalls (siehe pp. 404-407). Werden durch einen Abfall mehrere Veränderungen gleichzeitig bewirkt, so ist derjenige Grenzwert entscheidend, der das größte schädlich veränderte Meerwasservolumen ergibt.

Ein einmalig ins Meerwasser eingebrachter Abfall breitet sich vom Einbringungsort her nach allen Seiten hin aus. In manchen Fällen wird der Abfall gleichzeitig chemisch verändert, zum Beispiel wird Nitrit durch den Sauerstoff des Meerwassers zu Nitrat oxydiert. Das schädlich veränderte Meerwasservolumen wird nach dem Einbringen zunächst immer größer, erreicht dann einen Maximalwert und nimmt anschließend wieder $a b$, bis es den Wert Null erreicht hat. Der einige Zeit nach dem Einbringen eines Abfalls durchlaufene $\mathrm{Max}$ i ma $1 \mathrm{w}$ e $\mathrm{r}$ des schädlich veränderten Meerwasservolumens ist ein sehr gutes Maß für die Schädlichkeit eines einmalig ins Meerwasser eingebrachten Abfalls (siehe pp. 404-407). Für Oberschlagsrechnungen kann man den Maximalwert des schädlich veränderten Meerwasservolumens demjenigen Meerwasservolumen gleichsetzen, das erforderlich ist, um den betreffenden Abfall bis zur Ungefährlichkeit und Unschädlichkeit, das heißt bis zum entscheidenden Grenzwert, zu verdünnnen. Das mehrmalige Einbringen eines Abfalls brauchen wir hier nicht gesondert zu betrachten, weil man nicht mehrmals nacheinander einen Abfall in dasselbe Meerwasservolumen einbringen wird. Daher läßt sich das mehrmalige Einbringen eines Abfalls fast immer auf das einmalige Einbringen zurückführen. 


\title{
Veränderungen an der Meeresoberfläche
}

\begin{abstract}
Abfallstoffe, die leichter als Meerwasser sind, schwimmen an der Meeresoberfläche. Manche Abfälle, wie beispielsweise Polyaethylen, treiben sehr lange Zeir auf der Meeresoberfläche herum, bis sie irgendwo an der Küste angetrieben werden. Andere Abfälle verschwinden nach einiger Zeit von der Meeresoberfläche, weil sie absinken ( $z, B$. Holz), in Lösung gehen oder sich verflüchtigen ( $z, B$. die leichteren Fraktionen des Erdöls) oder chemisch verändert werden (z. B. Erdöl, das durch Mikroorganismen abgebaut wird).
\end{abstract}

\section{Veränderungen in der Luft über dem Meer}

Flüchtige Abfälle können die über dem Meer befindliche Luft verändern. So können beispielsweise organische Lösungsmittel, die ins Meer geschüttet werden, in die Luft übertreten und dort Menschen und Tiere belästigen oder schädigen. Eine andere Möglichkeit besteht darin, daß zunächst nicht flüchtige Abfallstoffe durch chemische Veränderungen in flüchtige Stoffe umgewandelt werden. So kann zum Beispiel Arsenat durch die Tätigkeit von Mikroorganismen in flüchtige organische ArsenVerbindungen überfuhrt werden. Hierdurch soll die sog. "Haffkrankheit“ hervorgerufen werden.

\section{EINFUHRUNG VON FUNF GEFAHRENKLASSEN FUR DAS EINBRINGEN VON ABFALLEN INS MEER}

Oberstes Gebot für das Einbringen von Abfällen ins Meer ist, daß die negativen Wirkungen der Abfälle auf Pflanzen und Tiere des Meeres und der Küstenländer, auf Schiffahrt, Fischerei, Sport und Tourismus und nicht zuletzt auf die Gesundheit des Menschen möglichst klein gehalten werden müssen.

Dieser Forderung kann man dadurch gerecht werden, daß man bestimmte $\mathrm{Ab}$ fälle, zum Beispiel Polyaethylen, vom Meer gänzlich fernhält. Andere Abfälle, wie größere Mengen Calciumarsenat, Natriumnitrit oder Chlorkohlenwasserstoffe, wird man nur in Gebieten mit sehr großen Wassertiefen versenken dürfen. Demgegenüber wird man relativ harmlose Abfälle, wie kleinere Mengen Schwefelsäure oder größere Mengen Eisenoxide oder Fäkalien, in ein Schelfmeer einbringen können, ohne nennenswerte negative Auswirkungen befürchten zu müssen.

Aufbauend auf dieser Erkenntnis wurde vom Verfasser im Jahre 1964 eine Klassifizierung von Abfällen und Meeresgebieten vorgeschlagen und ausgearbeitet. Diese Klassifizierung sieht eine Einteilung von Abfällen in vier Gefahrenklassen vor und gibt für jede Gefahrenklasse diejenigen Meeresgebiete (Küstengewässer, offene See mit $<1500 \mathrm{~m}$ Tiefe, offene See mit $>1500 \mathrm{~m}$ Tiefe, offene See mit $>3000 \mathrm{~m}$ Tiefe) an, in welche die betreffenden Abfälle eingebracht werden dürfen. Diese Klassifizierung ist das Kernstück eines Gutachtens ("Gutachten über die Verschmutzung der See durch industrielle Abfallstoffe"), das 1965 im Auftrage des Bundesministers für 
Verkehr der Bundesrepublik Deutschland vom Deutschen Hydrographischen Institut, der Bundesforschungsanstalt für Fischerei und der Bundesanstalt für Gewässerkunde angefertigt wurde.

Es sei hervorgehoben, daß die in dem Gutachten vorgenommene Einteilung in Gefahrenklassen nicht nur die Stoffe, sondern auch die Mengen berücksichtigt. Demgegenüber dürfte eine Einteilung allein nach Stoffen wenig sinnvoll sein. Das zeigt ein ganz einfaches Beispiel: Kaliumcyanid ist eine sehr gefährliche Substanz. Werden größere Mengen davon in ein Schelfmeer eingebracht, so ist mit Sicherheit eine nennenswerte Schädigung der Meeresorganismen zu erwarten. Dagegen wird eine sehr kleine Menge Kaliumcyanid nur einen entsprechend geringen oder gar keinen Schaden im Meer anrichten. Die Gefährlichkeit des Kaliumcyanids im Meer kann also je nach der zu beseitigenden Menge sehr verschieden sein.

Die ursprüngliche Einteilung der Abfälle in vier Gefahrenklassen enthielt eine Präambel. Darin wurde unter anderem festgelegt, daß Abfälle, deren Dichte kleiner als die des Meerwassers ist und die gleichzeitig im Meerwasser schwer löslich sind und durch Mikroorganismen nur langsam abgebaut werden können wie beispielsweise Polyaethylen, überhaupt nicht ins Meer eingebracht werden dürfen. Nach den Erfahrungen der letzten Jahre erscheint es zweckmäßig, für derartige Abfälle eine besondere Gefahrenklasse einzuführen. In diese Gefahrenklasse können dann auch solche Abfälle eingeordnet werden, deren Einbringung ins Meer aus anderen Gründen unverantwortlich erscheint.

Die neue Klassifizierung, die nunmehr fünf Gefahrenklassen umfaßt, ist in Tabelle 1 dargestellt: Am oberen Rand der Tabelle 1 sind die fünf Gefahrenklassen aufgetragen. Die linke Spalte unterscheidet vier verschiedene Arten von Meeresgebieten, und zwar Meeresgebiete mit $<20 \mathrm{~m}$ Tiefe, Meeresgebiete mit $20 \mathrm{~m}$ bis

Tabelle 1

Gefahrenklassen und Meeresgebiete für das Einbringen von Abfällen

\begin{tabular}{|lccccc|}
\hline Tiefen-Zonen & 1 & 2 & 3 & 4 & 5 \\
\hline Meeresgebiete mit $<20 \mathrm{~m}$ Tiefe & (ja) & nein & nein & nein & nein \\
Meeresgebiete mit 20-1000 m Tiefe & ja & (ja) & nein & nein & nein \\
Meeresgebiete mit 1000-4000 m Tiefe & ja & ja & (ja) & nein & nein \\
Meeresgebiete mit $>4000 \mathrm{~m}$ Tiefe & ja & ja & ja & (ja) & nein \\
\hline
\end{tabular}

$1000 \mathrm{~m}$ Tiefe, Meeresgebiete mit $1000 \mathrm{~m}$ bis $4000 \mathrm{~m}$ Tiefe und Meeresgebiete mit $>4000 \mathrm{~m}$ Tiefe. Im Innern der Tabelle 1 (Spalten 2 bis 6 und Zeilen 3 bis 6 ) ist die Frage beantwortet, in welche Meeresgebiete ein Abfall einer bestimmten Gefahrenklasse eingebracht werden darf. Die Frage ist mit „ja“, „(ja)“ oder "nein“ beantwortet; das "Ja" bedeutet, daß der betreffende Abfall unter Einhaltung allgemei- 
ner Bedingungen in das entsprechende Meeresgebiet eingebracht werden darf. Das "(Ja)“ bedeutet, daß beim Einbringen der Abfälle außer den allgemeinen Bedingungen auch noch spezielle Bedingungen einzuhalten sind.

$\mathrm{Zu}$ den „allgemeinen Bedingungen“ zählen beispielsweise folgende Vorschriften: (1) Abfälle, die wegen ihrer Gefährlichkeit in Meeresgebiete mit $>1000 \mathrm{~m}$ Tiefe eingebracht werden müssen, sind, soweit möglich, auf den Meeresboden zu versenken, und zwar so, daß die Abfälle während des Absinkens nicht in Lösung gehen. (2) In Gebieten, wo der Meeresboden dicht vor der Küste steil abfällt, ist beim Einbringen von Abfällen, die den Luftraum über dem Meer oder die Meeresoberfläche verändern, ein bestimmter Mindesabstand von der Küste einzuhalten. (3) Mit Abfällen gefüllte Fässer, Flaschen etc. dürfen nicht in solche Meeresgebiete versenkt werden, in denen Schleppnetzfischerei betrieben wird. (4) Feste, schwer lösliche Abfälle sind in flachen Küstengewässern so zu versenken, daß sie die Schiffahrt nicht gefährden.

Als „spezielle Bedingungen“ kann man zum Beispiel festlegen, daß ein Abfall nur an einer bestimmten Stelle ins Meer eingebracht werden darf, daß das Einbringen eines Abfalls auf einen längeren Zeitraum auszudehnen ist oder daß ein Abwasser während des Einbringens kräftig mit Meerwasser zu verdünnen ist.

Aus Tabelle 1 sind folgende Einzelheiten zu entnehmen: (1) In Meeresgebiete mit $<20 \mathrm{~m}$ Tiefe dürfen nur Abfälle der Gefahrenklasse 1 eingebracht werden und auch diese nur unter Einhaltung spezieller Bedingungen. (2) In Meeresgebiete mit $20 \mathrm{~m}$ bis $1000 \mathrm{~m}$ Tiefe dürfen nur Abfälle der Gefahrenklassen 1 und 2 eingebracht werden. Beim Einbringen von Abfällen der Klasse 2 sind spezielle Bedingungen einzuhalten. (3) In Meeresgebiete mit $1000 \mathrm{~m}$ bis $4000 \mathrm{~m}$ Tiefe dürfen nur Abfälle der Gefahrenklassen 1, 2 und 3 eingebracht werden. Beim Einbringen von Abfällen der Klasse 3 sind spezielle Bedingungen einzuhalten. (4) In Meeresgebiete mit $>4000 \mathrm{~m}$ Tiefe dürfen Abfälle der Gefahrenklassen 1, 2, 3 und 4 eingebracht werden. Beim Einbringen von Abfällen der Klasse 4 sind spezielle Bedingungen einzuhalten. (5) Abfälle der Gefahrenklasse 1 dürfen in alle Meeresgebiete eingebracht werden. Beim Einbringen in Meeresgebiete mit $<20 \mathrm{~m}$ Tiefe sind spezielle Bedingungen einzuhalten. (6) Abfälle der Gefahrenklasse 2 dürfen nur in Meeresgebiete mit $>20 \mathrm{~m}$ Tiefe eingebracht werden. Beim Einbringen in Meeresgebiete mit $20 \mathrm{~m}$ bis $1000 \mathrm{~m}$ Tiefe sind spezielle Bedingungen einzuhalten. (7) Abfälle der Gefahrenklasse 3 dürfen nur in Meeresgebiete mit $>1000 \mathrm{~m}$ Tiefe eingebracht werden. Beim Einbringen in Meeresgebiete mit $1000 \mathrm{~m}$ bis $4000 \mathrm{~m}$ Tiefe sind spezielle Bedingungen einzuhalten. (8) Abfälle der Gefahrenklasse 4 dürfen nur in Meeresgebiete mit $>4000 \mathrm{~m}$ Tiefe eingebracht werden und auch das nur unter Einhaltung spezieller Bedingungen. (9) Abfälle der Gefahrenklasse 5 dürfen überhaupt nicht ins Meer eingebracht werden.

Innerhalb der einzelnen Tiefen-Zonen sind die Abfälle nach Möglichkeit dort einzubringen, wo die hydrographischen Bedingungen (z. B. Strömung und Diffusion) am günstigsten sind, wo die Organismendichte am kleinsten ist und wo die Nutzung des Meeres durch den Menschen (z. B. Fischerei und Süßwassergewinnung) am geringsten ist.

Tabelle 1 gibt klare Auskunft darüber, ob ein Abfall einer bestimmten Gefahrenklasse in ein bestimmtes Meeresgebiet eingebracht werden darf oder nicht. Außer- 
dem ist in der Tabelle angegeben, ob beim Einbringen eines Abfalls ins Meer außer den allgemeinen Bedingungen auch spezielle Bedingungen eingehalten werden müssen.

Nunmehr soll die Einordnung der verschiedenen Abfälle in die einzelnen $\mathrm{Gef}$ ahrenklassen besprochen werden. Die Einordnung der Abfälle in die Gefahrenklassen richtet sich nach der Art, dem A us ma und der Dauer der Veränderungen, die von den Abfällen im Meer hervorgerufen werden. Im folgenden sind die Veränderungen zusammengestellt, auf Grund welcher die Abfälle in die einzelnen Gefahrenklassen einzuordnen sind. Für die Einordnung genügt jeweils eine der vier aufgeführten Veränderungen.

Veränderungen, welche die Einordnung von Abfällen in die Gefahren$\mathrm{k} l$ a s s e 1 bedingen: (1) Veränderungen am Meeresboden: Beim Versenken schwer löslicher Abfälle ins Meer wird am Meeresboden nur eine kleine Fläche $\left(<10^{5} \mathrm{~m}^{2}\right)$ von den Abfällen zugedeckt. (2) Veränderungen im Meerwasser: Durch das Einbringen der Abfälle ins Meer werden nur in einem kleinen Meerwasservolumen $\left(<10^{6} \mathrm{~m}^{3}\right)$ schädliche Veränderungen hervorgerufen. Bei kontinuierlichem Einbringen ist der zeitlich konstante Wert, bei einmaligem Einbringen der zeitliche Maximalwert des schädlich veränderten Meerwasservolumens ausschlaggebend (vgl. p. 400). (3) Veränderungen an der Meeresoberfläche: Da die Abfälle nicht schwimmen, wird die Meeresoberfläche durch das Einbringen der Abfälle nicht verändert. (4) Veränderungen in der Luft über dem Meer: Durch das Einbringen der Abfälle ins Meer werden in der über dem Meer befindlichen Luft keine Veränderungen hervorgerufen.

Veränderungen, welche die Einordnung von Abfällen in die Gefahren$\mathrm{k}$ l a s s e 2 bedingen: (1) Veränderungen am Mecresboden: Beim Versenken schwer löslicher Abfälle ins Meer wird am Meeresboden eine größere Fläche (10 bis $10^{7} \mathrm{~m}^{2}$ ) von den Abfällen zugedeckt. (2) Veränderungen im Meerwasser: Durch das Einbringen der Abfälle ins Meer werden in einem größeren Meerwasservolumen (10 ${ }^{6}$ bis $10^{8} \mathrm{~m}^{3}$ ) schädliche Veränderungen hervorgerufen. Bei kontinuierlichem Einbringen ist der zeitlich konstante Wert, bei einmaligem Einbringen der zeitliche Maximalwert des schädlich veränderten Meerwasservolumens ausschlaggebend (vgl. p. 400). (3) Veränderungen an der Meeresoberfläche: Durch das Einbringen der Abfälle ins Meer treten an der Meeresoberfläche nur geringe, sehr kurzzeitige Veränderungen ein. (4) Veränderungen in der Luft über dem Meer: Durch das Einbringen der Abfälle ins Meer werden in der über dem Meer befindlichen Luft nur geringe Veränderungen hervorgerufen.

Veränderungen, welche die Einordnung von Abfällen in die Gefahren $\mathrm{k} 1$ a s s e 3 bedingen: (1) Veränderungen am Meeresboden: Beim Versenken schwer löslicher Abfälle ins Meer wird am Meeresboden eine große Fläche $\left(>10^{7} \mathrm{~m}^{2}\right)$ von den Abfällen zugedeckt. (2) Veränderungen im Meerwasser: Durch das Einbringen der Abfälle ins Meer werden in einem relativ großen Meerwasservolumen (108 bis $10^{10} \mathrm{~m}^{3}$ ) schädliche Veränderungen hervorgerufen. Bei kontinuierlichem Einbringen ist der zeitlich konstante Wert, bei einmaligem Einbringen der zeitliche Maximalwert des schädlich veränderten Meerwasservolumens ausschlaggebend (vgl. p. 400). (3) Veränderungen an der Meeresoberfläche: Durch das Einbringen der Abfälle ins Meer treten an der Meeresoberfläche nur sehr kurzzeitige Veränderungen ein. (4) Ver- 


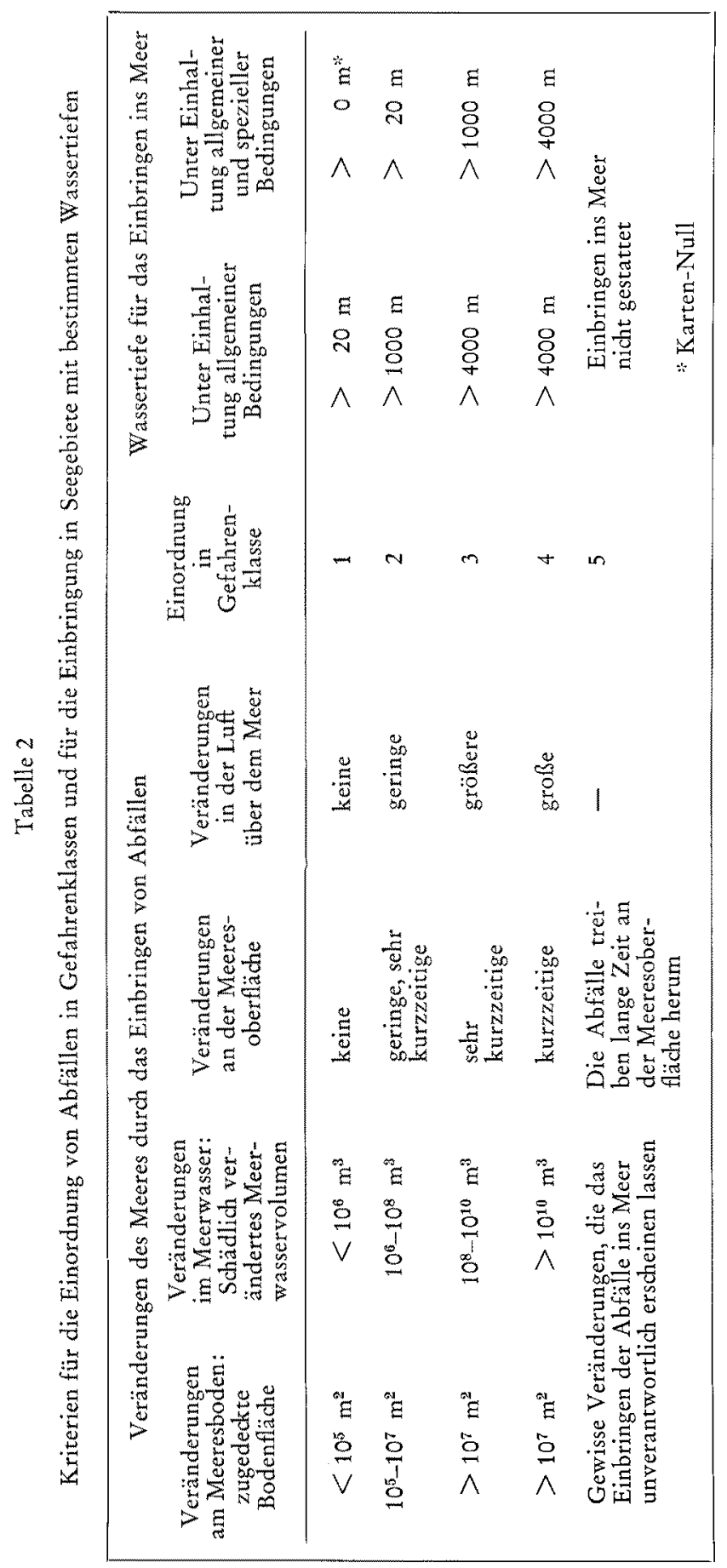


änderungen in der Luft über dem Meer: Durch das Einbringen der Abfälle ins Meer werden in der über dem Meer befindlichen Luft größere Veränderungen hervorgerufen.

Veränderungen, welche die Einordnung von Abfällen in die Gefahrenk la s se 4 bedingen: (1) Veränderungen am Meeresboden: Beim Versenken schwer löslicher Abfälle ins Meer wird am Meeresboden eine große Fläche $\left(>10^{7} \mathrm{~m}^{2}\right)$ von den Abfällen zugedeckt. (2) Veränderungen im Meerwasser: Durch das Einbringen der Abfälle ins Meer werden in einem großen Meerwasservolumen $\left(>10^{10} \mathrm{~m}^{3}\right)$ schädliche Veränderungen hervorgerufen. Bei kontinuierlichem Einbringen ist der zeitlich konstante Wert, bei einmaligem Einbringen der zeitliche Maximalwert des schädlich veränderten Meerwasservolumens ausschlaggebend (vgl. p. 400). (3) Veränderungen an der Meeresoberfläche: Durch das Einbringen der Abfälle ins Meer treten an der Meeresoberfläche nur kurzzeitige Veränderungen ein. (4) Veränderungen in der Luft über dem Meer: Durch das Einbringen der Abfälle ins Meer werden in der über dem Meer befindlichen Luft große Veränderungen hervorgerufen.

Veränderungen, welche die Einordnung von Abfällen in die Gefahrenk l a s s e 5 bedingen: (1) Veränderungen an der Meeresoberfläche: Die Abfälle treiben für längere oder lange Zeit an der Meeresoberfläche herum. (2) Sonstige Veränderungen des Meeres: Es treten andere Veränderungen ein, die das Einbringen der Abfälle ins Meer unverantwortlich erscheinen lassen.

Die hier aufgeführten Kriterien für die Einordnung von Abfällen in die verschiedenen Gefahrenklassen sind in Tabelle 2 noch einmal in gekürzter Form zusammengestellt. Daneben enthält Tabelle 2 auch die Angaben von Tabelle 1 darüber, ob und bei welchen Wassertiefen die Abfälle ins Meer eingebracht werden dürfen.

Das in Spalte 2 der Tabelle 2 aufgeführte schädlich veränderte Meerwasservolumen ist dasjenige Volumen, in dem durch das Einbringen von Abfällen Schäden auftreten können, weil ein bestimmter Grenzwert, zum Beispiel eine Grenztemperatur oder eine Grenzkonzentration (z. B. die Erträglichkeitsgrenze) über- oder unterschritten ist. Bei kontinuierlichem Einbringen von Abfällen ist der zeitlich konstante Wert, bei einmaligem Einbringen der zeitliche Maximalwert des schädlich veränderten Meerwasservolumens ausschlaggebend (vgl. p. 400).

Bei der Benutzung der Tabelle 2 sind folgende Regeln zu beachten: (1) Ergeben sich für einen Abfall auf Grund mehrerer Veränderungen nach dem Schema verschiedene Gefahrenklassen, so ist die höchste von diesen maßgebend. (2) Abfälle, die am Meeresboden $>10^{7} \mathrm{~m}^{2}$ zudecken, sind in Gefahrenklasse 3 einzuordnen. Die Gefahrenklasse 4 entfällt hier (siehe Spalte 1). (3) Ist das für die Aufnahme eines Abfalls bestimmte Meerwasser bereits vorbelastet, so ist dies bei der Berechnung des schädlich veränderten Meerwasservolumens zu berücksichtigen.

Zum Schluß an Hand der Tabelle 2 einige praktische Beispiele.

(a) $1000 \mathrm{t}$ Eisenoxid sollen ins Meer versenkt werden. Bei einer Schichtdicke von 5 bis $10 \mathrm{~cm}$ wird von dem Eisenoxid eine Fläche von $10^{4} \mathrm{~m}^{2}$ zugedeckt. Dieser Fall ist nach Tabelle 2 in Gefahrenklasse 1 einzuordnen.

(b) Meerwasserkonzentrat aus einer Meerwasserentsalzungsanlage soll kontinuierlich ins Meer eingeleitet werden. Das schädlich veränderte Meerwasser- 
volumen wird im stationären Zustand voraussichtlich $10^{5} \mathrm{~m}^{3}$ betragen. Dieser Fall ist nach Tabelle 2 in Gefahrenklasse 1 einzuordnen.

(c) Verdünnte Schwefelsäure soll kontinuierlich ins Meer eingeleitet werden. Das schädlich veränderte Meerwasservolumen wird im stationären Zustand voraussichtlich $10^{7} \mathrm{~m}^{3}$ betragen. Dieser Fall ist nach Tabelle 2 in Gefahrenklasse 2 einzuordnen.

(d) $1000 \mathrm{~m}^{3}$ verdünnte Schwefelsäure (20\% ig) sollen ins Meer eingebracht werden. Zur Verdünnung bis zur Unschädlichkeit ist etwa das $10^{4}$ fache Meerwasservolumen, also insgesamt ca. $10^{7} \mathrm{~m}^{3}$ Meerwasser erforderlich. Ungefähr von der gleichen Größe ist der zeitliche Maximalwert des schädlich veränderten Meerwasservolumens (vgl. p. 400). Dieser Fall ist nach Tabelle 2 in Gefahrenklasse 2 einzuordnen.

(e) 200 t Abfälle, die etwa 5 t lösliches Kupfer enthalten, sollen ins Meer versenkt werden. Zur Verdünnung bis zur Unschädlichkeit sind etwa $5 \cdot 10^{8} \mathrm{~m}^{3}$ Meerwasser erforderlich. Ungefähr von der gleichen Größe ist der zeitliche Maximalwert des schädlich veränderten Meerwasservolumens (vgl. p. 400). Dieser Fall ist nach Tabelle 2 in Gefahrenklasse 3 einzuordnen.

(f) Calciumarsenat-Abfälle, die $500 \mathrm{t}$ Arsen enthalten, sollen ins Meer versenkt werden. Der Arsen-Gehalt des natürlichen Seewassers liegt bei $10 \mathrm{mg} / \mathrm{m}^{3}$. Nehmen wir an, daß die Grenzkonzentration bei dem doppelten Wert der natürlichen Arsenkonzentration, also bei $20 \mathrm{mg} / \mathrm{m}^{3}$ liegt, so kann $1 \mathrm{~m}^{3}$ Meerwasser noch $10 \mathrm{mg}$ Arsen aufnehmen, ohne daß Schäden entstehen. Für 500 t Arsen sind demnach zur Verdünnung bis zur Unschädlichkeit $5 \cdot 10^{10} \mathrm{~m}^{3}$ Meerwasser exforderlich. Ungefähr von der gleichen Größe ist der zeitliche Maximalwert des schädlich veränderten Meerwasservolumens (vgl. p. 400). Dieser Fall ist nach Tabelle 2 in Gefahrenklasse 4 einzuordnen.

(g) 100 t Polyaethylenfolie sollen ins Meer eingebracht werden. Da Polyaethylen sehr lange auf dem Meer herumschwimmt, ist dieser Fall nach Tabelle 2 in Gefahrenklasse 5 einzuordnen.

\section{ZUSAMMENFASSUNG}

1. Die Veränderungen des Meeres, die durch das Einbringen von Abfällen ins Meer hervorgerufen werden können, werden besprochen.

2. Vier verschiedene Arten von Veränderungen werden unterschieden: (a) Veränderungen am Meeresboden, (b) Veränderungen im Meerwasser, (c) Veränderungen an der Meeresoberfläche, (d) Veränderungen in der Luft über dem Meer.

3. Als Maß für die Schädlichkeit schwer löslicher Abfälle, die auf den Meeresboden absinken, wird die von den Abfällen zugedeckte Fläche am Meeresboden benutzt.

4. Als Maß für die Schädlichkeit von Abfällen im Meerwasser wird das „schädlich veränderte Meerwasservolumen “ eingeführt. Das schädlich veränderte Meerwasservolumen ist dasjenige Volumen, in dem durch das Einbringen von Abfällen Schäden auftreten können, weil ein bestimmter Grenzwert, beispielsweise eine Grenztemperatur oder eine Grenzkonzentration (z. B. die Erträglichkeits- 
grenze), über- oder unterschritten ist. Bei kontinuierlichem Einbringen von Abfällen ist der zeitlich konstante Wert, bei ein- oder mehrmaligem Einbringen der zeitliche Maximalwert des schädlich veränderten Meerwasservolumens ausschlaggebend. Für Oberschlagsrechnungen kann man den zeitlichen Maximalwert des schädlich veränderten Meerwasservolumens demjenigen Meerwasservolumen gleichsetzen, das erforderlich ist, um den betreffenden Abfall bis zur Ungefährlichkeit und Unschädlichkeit zu verdünnen.

5. Als oberstes Gebot für das Einbringen von Abfällen ins Meer wird die Notwendigkeit herausgestellt, die negativen Wirkungen der Abfälle auf Pflanzen und Tiere des Meeres und der Küstenländer, auf Schiffahrt, Fischerei, Sport und Tourismus und nicht zuletzt auf die Gesundheit des Menschen so klein wie möglich zu halten.

6. Unter Berücksichtigung dieses Gebotes wird eine Klassifizierung von Abfällen und Meeresgebieten vorgeschlagen.

7. Die vorgeschlagene Klassifizierung teilt die Abfälle unter Berücksichtigung von Stoff und Menge in fünf Gefahrenklassen ein und gibt an, ob und bei welchen Wassertiefen die Abfälle ins Meer eingebracht werden dürfen.

8. Für die Einordnung von Abfällen in die einzelnen Gefahrenklassen werden Kriterien angegeben. Diese Kriterien berücksichtigen Art, Ausmaß und Dauer der durch die Abfälle im Meer hervorgerufenen Veränderungen.

9. Die Kriterien für die Einordnung von Abfällen in die einzelnen Gefahrenklassen und die Angaben darïber, ob und bei welchen Wassertiefen die Abfälle ins Meer eingebracht werden dürfen, sind in Form einer Tabelle zusammengestellt.

10. An Hand von praktischen Beispielen wird die vorgeschlagene Klassifizierung erläutert.

\section{Dikussion im Anschluß an den Vortrag WeICHART}

KINNE: Sie haben das Problem der Einbringung von Abfallstoffen ins Meer eingehend durchdacht und ein Schema für 5 Gefahrenklassen entwickelt. Dieses Schema ist klar konzipiert und übersichtlich gegliedert. Es trägt dem Bedürfnis nach einer ersten gedanklichen Durchdringung Rechnung. Leider liegen die Dinge in der freien Natur nicht immer ganz so einfach. Sie haben als Kriterien unter anderem die Größe des durch Abfallstoffe bedeckten Bodenareals und die Wassertiefe benutzt. Wenn ein Bodenareal von $10^{4} \mathrm{qm} \mathrm{z}$. B. mit Eisenhydroxid bedeckt wird, dann ist es für die Festsetzung der Gefahrenklasse zweifellos nicht gleichgültig, ob dieses Gebiet in einer kaum besiedelten "Wüste" liegt oder ob es sich dabei um Laichgründe des Herings handelt. Thre Gefahrenklasse 2 umfaßt Wassertiefen von 20 bis $1000 \mathrm{~m}$. Hier muß sicherlich noch differenziert werden. Gerade im flachen Litoralbereich liegen Laich- und Auf wuchsgebiete zahlreicher Meeresorganismen. Von diesen Gebieten aus erhalten viele als Adultus im freien Meer lebende Organismen ständig „Nachschub“. Gleichzeitig stellen diese Gebiete vielfach auch die "Speisekammer" für zahlreiche räuberische Formen des offenen Meeres dar. Bei der Festlegung von Tiefenstufen müssen also auch horizontale biologische Transport- und Austauschvorgänge mitberücksichtigt werden. Ihr Schema bedarf daher der Ergänzung durch die lokale biologische Situation. Wenn ich Sie recht verstanden habe, gehen Sie ferner bei Ihren 5 Gefahrenklassen von idealen Vermischungsvorgängen und von reinem Meerwasser aus. Wir wissen, daß die Vermischungsvorgänge in starkem Maß von der örtlichen hydrographischen Situation abhängen; Herr ToMCZAK hat $j a$ in seinem Einführungsvortrag schon darauf hingewiesen. Insbesondere in den Küstenbereichen der Nordsee ist das Meerwasser häufig bereits 
verunreinigt, bevor das von Thnen vorgeschlagene Schema zur Anwendung kommen kann. Bei der Ermittlung der Gefahrenklasse müßte das schon vorher bestehende Ausmaß der Abwasserbelastung daher in Rechnung gestellt werden.

WeIchart: Herr Professor KInNe, ich gebe Ihnen völlig recht. Die Praxis ist erheblich komplizierter als dieses Schema. Dieses Schema soll aud nur einen äußeren Rahmen geben, um Gerechtigkeit walten zu lassen gegenüber den verschiedenen Industriekreisen, die bei uns um Zustimmung für das Einbringen yon Abfällen ins Meer nachsuchen. Nun zu den biologischen Aspekten: Diese sind in der Klassifizierung implizit enthalten, und zwar in der Erträglichkeitsgrenze unter Mitberücksichtigung der Anreicherungsmöglichkeiten in den Organismen.

Kinne: Die Sache hat nur den Schönheitsfehler, daß wir über diese biologischen Dinge noch wenig wissen und daß Erträglichkeitsgrenzen allein zur Beurteilung der Situation nicht ausreichen.

Weichart: Das wird die zukünftige Aufgabe der Biologen sein.

Toмczak: Ich möchte noch eine Ergänzung zu der Bemerkung von Professor Kinne geben. Natürlich müssen die hydrographischen Bedingungen der Verseuchungsstelle sorgfältig mit berücksichtigt werden. Zum Beispiel umfaßt die Gefahrenklasse 2 von Dr. Werchart Tiefen $z$ wischen 20 und $1000 \mathrm{~m}$, das heißt wir haben eine Wahl zwischen der Umgebung von Helgoland bis zum Kontinentalsockel zum Europäischen Nordmeer oder dem Atlantik in der Biskaya. Wie mein Vortrag über die Klassifikation industrieller Abfallstoffe auf diesem Symposion zeigen sollte, sind auf diesem Gebiet starke hydrographische Verschiedenheiten, die uns eine entsprechende Auswahl gestatten.

Wetchart: Wie ich schon in meinem Vortrag vorgeschlagen habe, sollte man unter den „speziellen Bedingungen“ für das Einbringen von Abfällen ins Meer vorschreiben können, daß ein gegebener Abfall innerhalb einer bestimmten Tiefenstufe nur an einer bestimmten Stelle ins Meer eingebracht werden darf. Bei der Auswahl der Versenkungsstelle wird man selbstverständlich die hydrographischen Bedingungen und die Nutzung des Meeres durch den Menschen berücksichtigen.

Rheinheimer: Mir scheint die Zone 2 (20 bis $1000 \mathrm{~m}$ Tiefe) im Hinblick auf unsere eigenen Gewässer in Nord- und Ostsee etwas zu weit gefaßt. In unserem Teil der westlichen Ostsee beispielsweise sind die Seegebiete mit mehr als $20 \mathrm{~m}$ Tief auf schmale Rinnen beschränkt, die sehr leicht überlastet werden können - vor allem, wenn es sich un unlösliche Abfälle handelt. Ahnlich ist es auch in der Deutschen Bucht. Was wird geschehen, wenn einzelne Staaten ihre Hoheitsgrenzen ausdehnen und den Festlandsockel aufteilen? Besteht dann nicht die Gefahr, daß einzelnen Industriestaaten dann nur noch sehr begrenzte Meeresgebiete für die Abfallbeseitigung zur Verfügung stehen?

WEICHART: Sie haben recht, die Tiefenstufe von 20 bis $1000 \mathrm{~m}$ erscheint recht groß. Der Sinn dieser Tiefenstufenbegrenzung ist folgender: Alle gefährlichen Abfälle sollen von Gebieten, in denen Fischerei betrieben werden kann oder betrieben wird, ferngehalten werden. Die größte Tiefe, in der heute gefischt wird, liegt bei ca. $750 \mathrm{~m}$. Daher die obere Begrenzung von $1000 \mathrm{~m}$ ( $250 \mathrm{~m}$ Sicherheitsabstand). Auf der anderen Seite sind die Abfälle der Gefahrenklasse 2 nicht so harmlos, daß man sie unmittelbar vor der Küste ins Meer einbringen könnte. Daher die untere Begrenzung der Tiefenstufe von $20 \mathrm{~m}$ bzw. bei Steilabfall des Meeresbodens vor der Küste die Vorschrift, einen bestimmten Mindestabstand yon der Küste einzuhalten.

ToMczaK: Eine Ergänzung zu der Frage von Herrn RherNHermer. Es scheint mir eine Verwechslung vorzuliegen. Wir müssen klar unterscheiden: (a) Hoheitsgewässer; diese erstrecken sich auf die Zone bis $3 \mathrm{sm}$ vor der Küste eines Landes. (b) Fischereihoheit; hier erheben die verschiedenen Staaten Ansprüche auf einen Streifen von 3 bis $12 \mathrm{sm}$, einige bis zu $200 \mathrm{sm}$. Diese noch nicht allgemein anerkannten Ansprüche beziehen sich aber nur auf die Ausübung der Fischerei. (c) Rechte zur Ausbeutung der Bodenschätze; dies sind durch die Genfer Konvention über den Kontinentalschelf geregelt. Nach bestimmten Regeln ist hierin der Meeresboden bis $200 \mathrm{~m}$ Tiefe aufgeteilt. Dieses Recht bedeutet aber keine Hoheitsansprüche, die die Möglichkeit von Ausbringen von Abfallstoffen außerhalb der 3-sm-Zone beeinflussen könnten. 
WeBER: Für Substanzen, die in Meerwasser löslich oder dispergierbar sind, wird durch das vorgeschlagene Verfahren angestrebt, in dem vorgesehenen Meeresgebiet mit der Substanz unterhalb einer bestimmten Grenzkonzentration, und zwar der Schädlichkeitsgrenze für Meeresorganismen zu bleiben. Ahnlich geht man auch zur Luftreinhaltung der Luft bei der Begrenzung einer Emission an luftfremden Stoffen vor. Die Substanz muß zur Einhaltung der geforderten Bedingungen durch geeignete technische Maßnahmen auf eine ausreichende Anfangsverdünnung gebracht werden. Bei Substanzen, die nur langsam in Lösung gehen, z. B. Arsen aus Arsenverbindungen, bietet die vorgeschlagene Einteilung sehr viel Sicherheit. Die betreffende Substanz wird die als Grenze angesetzte Konzentration für homogene Verteilung nie erreichen.

Koolen: In the Netherlands there are some sewage outfalls in the sea, and some others will perhaps be constructed in the future. Only sewage water may be discharged through them. There have been investigations done with Rhodamine B about the dilution of the discharged water in the sea at the proposed outfall places. It proved to be that the dilution in the sea was big indeed, but beyond a distance of some kilometers to the outfall the dilution increases very slowly. This depends on the amount of already mixed water that must be mixed further and that becomes bigger and bigger. So it proved to be best to calculate with the initial dilution only, and not with the dilution in the sea at greater distances. The initial dilution pattern covers an area of several hectares. The border of this area may be defined by the fact that there the dilution can be measured in a representative way. In this way only the turbulent area of initial dilution at the outfall will be "given up", as being harmful to the marine life. Within this area dilutions may be reached that are sufficient for many cases, except for the discharge of, for example, persistent insecticides. This means, in fact, that discharge of these materials will be forbidden. The dilution that occurs in the sea itself, on greater distances, will be an extra safety.

Weichart: Die Diffusion ist an den verschiedenen Stellen des Meeres sehr unterschiedlich. In ungünstigen Fällen ist die Diffusion nur gering. Dann dauert die Verdünnung eines einmalig ins Meer eingebrachten Abfalls bis zur Unschädlichkeit entsprechend lange. Bei kontinuierlichem Einleiten eines Abwassers ins Meer ist dann das schädlich veränderte Meerwasservolumen sehr groß. Uberschreitet die Größe des schädlich veränderten Meerwasservolumens eine bestimmte Grenze (vgl. Tabelle 2), so ist das Einleiten des betreffenden Abwassers an der vorgesehenen Stelle nidht mehr vertretbar. In der offenen Nordsee darf man im allgemeinen mit einer relativ großen Diffusion rechnen. Entsprechende Diffusionsversuche mit Rhodamin B sind hier in den letzten Jahren, teilweise in Zusammenarbeit mit anderen Nordseeanliegerstaaten, vom Deutschen Hydrographischen Institut Hamburg durchgeführt worden.

Simpson: I was most interested in this paper but consider that it is not realistic to make a rigid classification of this sort for administrative purposes. It does not, in my view, take into sufficient account the great differences between different coastal areas around the $20 \mathrm{~m}$ depth with regard to their capacity to receive pollutants without doing harm. Any decision on where a pollutant can be discharged must take into account the local topography, hydrography and fisheries resources.

WEICHART: Die vorgeschlagene Klassiffierung sieht vor, dẩ man innerhalb der verschiedenen Tiefenstufen den Ort des Einbringens vorschreiben kann. Bei der Auswahl der Versenkungsstelle muß man selbstverständlich die topographischen und hydrographischen Bedingungen sowie die Nutzung des Meeres berücksichtigen. 